\title{
Stage IB
}

National Cancer Institute

\section{Source}

National Cancer Institute. Stage IB. NCI Thesaurus. Code C27976.

Invasive cancer confined to the original anatomic site of growth without lymph node involvement. The definition of stage IB depends on the particular type of cancer that it refers to; for example, for breast cancer, stage IB is defined as follows: (TO, N1 mi, M0); (T1, N1 mi, M0). T0: No evidence of primary tumor. T1: Tumor $20 \mathrm{~mm}$ or less in greatest dimension. T1 includes T1mi. T1 mi: Tumor $1 \mathrm{~mm}$ or less in greatest dimension. N1 mi: Nodal micrometastases. M0: No clinical or radiog raphic evidence of distant metastasis. MO includes MO(i+); for bone cancer, stage IB is defined as follows: (T2, NO, MO, G1, G2, GX); (T3, N0, M0, G1, G2, GX). T2: T umor more than $8 \mathrm{~cm}$ in greatest dimension. T3: Discontinuous tumors in the primary bone site. N0: No regional lymph node metastasis. M0: No distant metastasis. G1: Well differentiated-low grade. G2: Moderately differentiated-low grade. GX: Grade cannot be assessed. (partially adapted from AJCC 7th ed.) 